\title{
Adaptation of Open Component-Based Systems
}

\author{
Pascal Poizat ${ }^{1,2}$ and Gwen Salaün ${ }^{3}$ \\ ${ }^{1}$ IBISC FRE 2873 CNRS - University of Evry Val d'Essonne, France \\ ${ }^{2}$ ARLES project, INRIA Rocquencourt, France \\ pascal.poizat@inria.fr \\ ${ }^{3}$ Department of Computer Science, University of Málaga, Spain \\ salaun@lcc.uma.es
}

\begin{abstract}
Software adaptation aims at generating software pieces called adaptors to compensate interface and behavioural mismatch between components or services. This is crucial to foster reuse. So far, adaptation techniques have proceeded by computing global adaptors for closed systems made up of a fixed set of components. This is not satisfactory when the systems may evolve, with components entering or leaving it at any time, e.g., for pervasive computing. To enable adaptation on such systems, we propose tool-equipped adaptation techniques for the computation of open systems adaptors. Our proposal also support incremental adaptation to avoid the computation of global adaptors.
\end{abstract}

\section{Introduction}

Compared to hardware components, software components (or services) are seldom reusable as is due to possible mismatch that may appear at different levels [10]: signature, behaviour, quality of service and semantics. Once detected, mismatch has to be corrected. However, it is not possible to impact directly on the components source due to their black-box nature. Software adaptation [2710] aims at generating, as automatically as possible, pieces of software called adaptors which are used to solve mismatch in a non intrusive way. To this purpose, model-based adaptation techniques base upon behavioural component interface descriptions and abstract properties of the adaptation called adaptation contracts or mappings. Dedicated middleware 2] can be used to put the adaptation process into action once an adaptor model (or implementation) has been obtained, but this is out of scope here.

Existing (global) adaptation approaches [27/21/4/811] proceed by generating a global adaptor for the whole system which is seen as a closed one. First of all this is costly. Moreover, when a component uses a service which does not relate through mapping to the other components' services, then either its use is prevented by adaptation (to avoid deadlock), or it is made internal (related events sent by the component are absorbed by the adaptor). Taking into account that new components, and hence new services, may be available in the future is not possible. Global adaptation approaches suffer from the fact that the adaptor has to be computed each time something changes in the system and are therefore not efficient in contexts such as pervasive systems [20], where services are not 
fixed or known from scratch. They may evolve, e.g., depending on the mobility of the user - moving around, different services are discovered and may be used, or on connectivity or management issues - some services may be temporary or definitely unavailable. In this paper we address these issues by extending a previous work for the adaptation of closed systems [11] in order to support (i) the adaptation of open systems and accordingly, (ii) an incremental process for the integration and adaptation of open software components. The definitions and algorithms we present have been implemented in Adaptor 11, our tool for model-based adaptation.

The paper is structured as follows. Section 2 presents our open systems component model and our adaptation techniques for such systems. In Section 3 we present the incremental adaptation of open systems, addressing the addition and the removal of components. Incremental adaptation has an added value at design-time, where the integration of components is known to be a difficult task, which gets worse when components have not been designed altogether from the beginning and therefore when adaptation connectors are needed. This typical use of the incremental adaptation of open systems is illustrated in Section 4. We end with comparison of related work and concluding remarks.

\section{Open Systems Adaptation}

In this section we address the adaptation of open systems. We first recall a formal model for basic sequential components originating from [11. Then we define an open composition model on top of it thanks to the definition of (i) compositional vectors, (ii) open synchronous product and (iii) open component-based systems and their semantics. In a second step, our adaptation algorithms are presented.

\subsection{Components}

Alphabets, the basis for interaction, correspond to an event-based signature. An alphabet $A$ is a set of service names, divided in provided services, $A^{\text {? }}$ (elements denoted as $e$ ?), required services, $A^{!}$(elements denoted as $e$ !) and internal actions (denoted with $\tau$ ). The mirror operation on an alphabet element is defined as $\overline{e ?}=e !, \overline{e !}=e ?$, and $\bar{\tau}=\tau$. Moreover, for an alphabet $A, \bar{A}=\{\bar{e} \mid e \in A\}$.

Component interfaces are given using a signature and a behavioural interface. A signature is a set of operation profiles as in usual component IDLs. This set is a disjoint union of provided operations and required operations. Behavioural interfaces are described in a concise way using a sequential process algebra, sequential CCS: $\mathrm{P}::=0 \mid \mathrm{a}$.P | a!.P | $\tau . \mathrm{P} \mid \mathrm{P} 1+\mathrm{P} 2$ | A, where 0 denotes termination, a?.P (resp. a!.P) a process which receives a (resp. sends it) and then behaves as P, $\tau$.P a process which evolves with the internal action $\tau$ (also denoted using tau in figures) and behaves as P, P1+P2 a process that acts either as $\mathrm{P} 1$ or $\mathrm{P} 2$, and $\mathrm{A}$ the call to a process defined by an equation $\mathrm{A}=\mathrm{P}$, enabling recursion. The CSS notation is extended using tags to support the definition of initial $([i])$ and final states $([f]) .0$ and $0[f]$ are equivalent. In order to define adaptation algorithms, we use the process algebra operational semantics 
to retrieve Labelled Transition Systems (LTS) from the interfaces, i.e., tuples $\langle A, S, I, F, T\rangle$ where $A$ is the alphabet (set of communication events), $S$ is the set of states, $I \in S$ is the initial state, $F \subseteq S$ is the set of final states, and $T \subseteq S \times A \times S$ are the transitions. The alphabet of a component LTS is built on this component's signature. This means that for each provided operation $p$ in the signature, there is an element $p$ ? in the alphabet, and for each required operation $r$, an element $r$ !.

\subsection{Open Component Systems}

Vectors are an expressive mechanism to denote communication and express correspondences between events in different processes. In this work vectors are extended to take into account open systems and keep track of their structuring. For this purpose, vectors are defined with reference to an (external) alphabet which relates component events to composite systems external interfaces (see Defs. 3 and 4, below).

Definition 1 ((Compositional) Vector). A compositional vector (or vector for short) $v$ for a set of LTSs $L_{i}=\left\langle A_{i}, S_{i}, I_{i}, F_{i}, T_{i}\right\rangle, i \in\{1, \ldots, n\}$ and an (external) alphabet $A_{\text {ext }}$ is an element of $A_{\text {ext }} \times\left(A_{1} \cup\{\varepsilon\}\right) \times \ldots \times\left(A_{n} \cup\{\varepsilon\}\right)$. Such a vector is denoted $e:\left\langle l_{1}, \ldots, l_{n}\right\rangle$ where $e \in A_{\text {ext }}$ and for every $i$ in $\{1, \ldots, n\}, l_{i} \in A_{i} \cup\{\varepsilon\} . \varepsilon$ is used in vectors to denote a component which does not participate in a communication.

The definition of an open synchronous product yields a tree-shaped structure for labels which makes it possible to keep trace of the structuring of composite components. When needed we may restrict to the observable part of labels, defined as $\operatorname{obs}\left(e:\left\langle l_{1}, \ldots, l_{n}\right\rangle\right)=e$. Moreover, labels of simple LTS can be related to composite ones using $l:\langle l\rangle$ for any label $l$.

Definition 2 (Open Synchronous Product). The open synchronous product of $n$ LTSs $L_{i}, i \in\{1, \ldots, n\}$ with reference to a set of vectors $V$ (defined over these LTSs and an external alphabet $\left.A_{\text {ext }}\right)$ is the $\operatorname{LTS} \Pi\left(\left(L_{1}, \ldots, L_{n}\right), A_{\text {ext }}, V\right)=$ $\langle A, S, I, F, T\rangle$ such that: $A=A_{\text {ext }} \times A_{1} \times \ldots \times A_{n}, S=S_{1} \times \ldots \times S_{n}, I=$ $\left(I_{1}, \ldots, I_{n}\right), F=F_{1} \times \ldots \times F_{n}$, and $T$ contains a transition $\left(\left(s_{1}, \ldots, s_{n}\right), e\right.$ : $\left.\left\langle a_{1}, \ldots, a_{n}\right\rangle,\left(s_{1}^{\prime}, \ldots, s_{n}^{\prime}\right)\right)$ iff there is a state $\left(s_{1}, \ldots, s_{n}\right)$ in $S$, there is a vector $e:\left\langle l_{1}, \ldots, l_{n}\right\rangle$ in $V$ and for every $i$ in $\{1, \ldots, n\}$ :

- if $l_{i}=\varepsilon$ then $s_{i}^{\prime}=s_{i}$ and $a_{i}=\varepsilon$,

- otherwise there is a transition $\left(s_{i}, a_{i}, s_{i}^{\prime}\right)$ with obs $\left(a_{i}\right)=l_{i}$ in $T_{i}$.

Remark. In practice, we reduce $S$ (resp. $F$ ) to elements of $S$ (resp. $F$ ) which are reachable from $I$ using $T$.

Example 1. Let us suppose we have two LTSs, $L_{1}$ and $L_{2}$, with one transition each: $\left(I_{1}, a\right.$ ?, $\left.S_{1}\right)$ for $L_{1}$ and $\left(I_{2}, b\right.$ !, $\left.S_{2}\right)$ for $L_{2}$. Different sets of vectors may express different communication semantics:

- $\{\tau:\langle a ?, b !\rangle\}$ (services $a$ ? and $b$ ! being synchronised) will produce a product LTS with a single transition: $\left(\left(I_{1}, I_{2}\right), \tau:\langle a ?, b !\rangle,\left(S_{1}, S_{2}\right)\right)$; 
- $\{a ?:\langle a ?, \varepsilon\rangle, b !:\langle\varepsilon, b !\rangle\}$ (services $a ?$ and $b$ ! left open to the environment) will produce a product LTS with four transitions:

$\left(\left(I_{1}, I_{2}\right), a ?:\langle a ?, \varepsilon\rangle,\left(S_{1}, I_{2}\right)\right),\left(\left(I_{1}, I_{2}\right), b !:\langle\varepsilon, b !\rangle,\left(I_{1}, S_{2}\right)\right)$ $\left(\left(S_{1}, I_{2}\right), b !:\langle\varepsilon, b !\rangle,\left(S_{1}, S_{2}\right)\right),\left(\left(I_{1}, S_{2}\right), a ?:\langle a ?, \varepsilon\rangle,\left(S_{1}, S_{2}\right)\right)$.

If we take this second case into account and make a product with an LTS $L_{3}$ with two transitions, $\left(I_{3}, c\right.$ !,$\left.S_{3}\right)$ and $\left(S_{3}, d ?, S_{3}^{\prime}\right)$, and vectors $\{\tau:\langle a ?, c !\rangle, \tau$ : $\langle b !, d ?\rangle\}$, we get a product LTS with two transitions:

$\left(\left(\left(I_{1}, I_{2}\right), I_{3}\right), \tau:\langle a ?:\langle a ?, \varepsilon\rangle, c !\rangle,\left(\left(S_{1}, I_{2}\right), S_{3}\right)\right)$,

$\left(\left(\left(S_{1}, I_{2}\right), S_{3}\right), \tau:\langle b !:\langle\varepsilon, b !\rangle, d ?\rangle,\left(\left(S_{1}, S_{2}\right), S_{3}^{\prime}\right)\right)$.

Composites denote sets of hierarchical connected open components.

Definition 3 (Composite (or Open Component System)). A composite is a tuple $\left\langle C, A_{\text {ext }}, B_{\text {int }}, B_{\text {ext }}\right\rangle$ where:

- $C$ is a set of component instances, i.e., an Id-indexed set of LTS $L_{i}, i \in I d$ (Id usually corresponds to the integers $\{1, \ldots, n\}$ ),

- $A_{\text {ext }}$ is an (external) alphabet,

- $B_{\mathrm{int}}$ is a set of vectors, with each vector $e:\left\langle l_{1}, \ldots, l_{n}\right\rangle$ in $B_{\mathrm{int}}$ being such that $e=\tau$, there is some $i$ in $\{1, \ldots, n\}$ such that $l_{i} \neq \varepsilon$ and there is at most one $j$ in $\{1, \ldots, n\} \backslash\{i\}$ such that $l_{j} \neq \varepsilon . B_{\text {int }}$ denotes internal (hidden) bindings between the composite sub-components, When clear from the context, such vectors can be denoted as couples $\left(l_{i}, l_{j}\right)$,

- $B_{\text {ext }}$ is a set of vectors, with each vector $e:\left\langle l_{1}, \ldots, l_{n}\right\rangle$ in $B_{\text {ext }}$ being such that $e \neq \tau$, there is some $i$ in $\{1, \ldots, n\}$ such that $l_{i} \neq \varepsilon$, and for every $k$ in $\{1, \ldots, n\} \backslash\{i\}, l_{k}=\varepsilon$. B $B_{\text {ext }}$ denotes external bindings between the composite sub-components and the composite interface itself. When clear from the context, such vectors can be denoted as couples $\left(e, l_{i}\right)$.

Our structure of composites supports (through model transformation) existing hierarchical ADLs such as the Fractal one 9] or UML 2.0 component diagrams [17. Note that with reference to these models we have an exact correspondence between their notions of component ports and component interfaces in what we call alphabets. Our model for bindings is more expressive than the Fractal ADL or UML 2.0 ones as we enable bindings between services with different names, which is mandatory to support adaptation.

Example 2. Let us take a component system described in an ADL (Fig. 1) where a batch processing client interacts with a database server to perform SQL requests. Our graphical notation is inspired from Fractal ADL, yet a textual notation is also supported. This model can be transformed into the following composite structure:

$\langle\{$ Client,SQLServer $\},\{$ launch?,exitCode! $\}$, $\{(\log !$, id? $),($ request!,sqlQuery?), (reply?,sqlValues!), (reply?,sqlError!), (end!, $\varepsilon)\}$, $\{($ launch?,run?), (exitCode!,exitCode!) $\}\rangle$.

Open synchronous product is used to give a formal semantics to composites. 


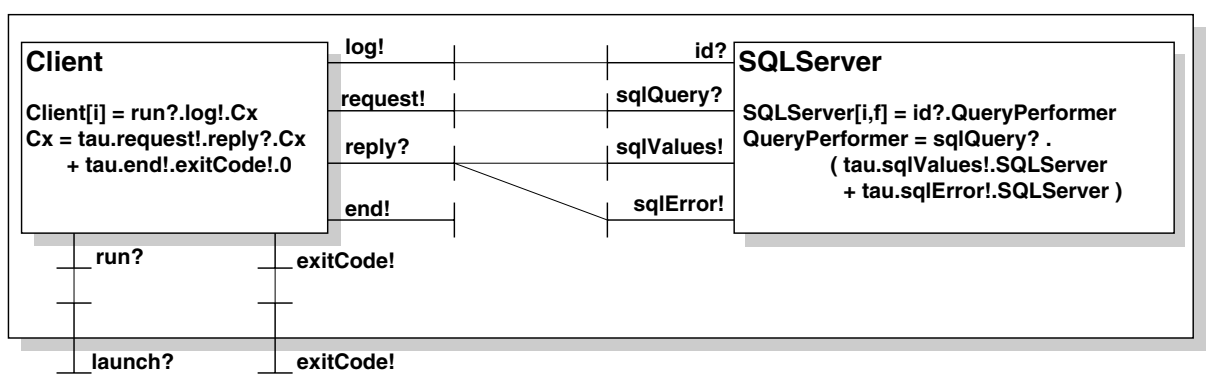

Fig. 1. The Client and SQLServer Example Architecture

Definition 4 (Composite Semantics). The semantics of a composite $\mathcal{C}=$ $\left\langle C, A_{\text {ext }}, B_{\text {int }}, B_{\text {ext }}\right\rangle$ is the $\operatorname{LTS} L(\mathcal{C})=\Pi\left(C, A_{\text {ext }}, \mathcal{V}\right)$ with $\mathcal{V}=B_{\text {int }} \cup B_{\text {ext }} \cup B_{\tau}$ where $B_{\tau}=\bigcup_{i \in\{1, \ldots, n\}}\left\{b_{\tau, i}\right\}$ and $b_{\tau, i}=\tau:\left\langle l_{1}, \ldots, l_{n}\right\rangle$ where $l_{i}=\tau$ and $l_{k}=\varepsilon$ for every $k$ in $\{1, \ldots, n\} \backslash\{i\}$.

In presence of several hierarchical levels (composites of composites), composite sub-components are first translated into LTSs using their semantics (Def. 4), e.g., the semantics of a composite $\mathcal{C}=\left\langle\left\{C_{1}, \ldots, C_{n}\right\}, A_{\text {ext }}, B_{\text {int }}, B_{\text {ext }}\right\rangle$ where some $C_{i}$ is a composite can be obtained replacing $C_{i}$ by $L\left(C_{i}\right)$ in $\mathcal{C}$.

\subsection{Mismatch and Mappings}

Composition correctness is defined in the literature [10] either at the composition model level - using deadlock freedom - or at the components' protocols level using compatibility or substitutability notions. As we want to use compositions in finding ways to correct mismatching components, we rely on the first approach. States without outgoing transitions are legal if they correspond to final states of the composed components. Therefore, we define deadlock (and hence mismatch) for a composite with a semantics $\langle A, S, I, F, T\rangle$ as a state $s \in S$ of the composition which has no outgoing transition $\left(\nexists\left(s, l, s^{\prime}\right) \in T\right)$ and is not final $(s \notin F)$. A deadlock is a state of the composition in which respective component protocols are incompatible, due to signature and/or behavioural mismatch. Note that if the former one can be solved using correspondences and renaming, the latter one requires more subtle techniques. This is also the case when correspondences evolve over time (e.g., in Example 4 below, id? in SQLServer corresponds first to log! in Client and later on to nothing).

Example 3. Let us get back to the composite presented in Example2, Mismatch in the example is due first to mismatching names. Moreover, even with an agreement on the service names, the fact that the client works in a connected mode (sending its log only once and disconnecting with end) while the server works in a non connected mode (requiring an id at each request) will also lead to behavioural mismatch after the first request of the client has been processed by the server. 
We propose regular expressions of open vectors as the means to express adaptation contracts. A regular expression (or regex for short) over some basic domain $\mathcal{D}$ is the set of all terms build on: $d$ (ATOM), R1.R2 (SEQUENCE), $R 1+R 2$ (CHOICE), $R 1 *$ (ITERATION) and $N$ (USE), with $d \in \mathcal{D}, R 1$ and $R 2$ being regular expressions, and $N$ being an identifier referring to a regex definition $N=R$. Such definitions can be used to structure regex but we forbid recursive definitions for operational reasons.

Definition 5 ((Adaptation) Mapping). An adaptation mapping (or mapping for short) for a composite $\mathcal{C}=\left\langle C, A_{\text {ext }}, B_{\text {int }}, B_{\text {ext }}\right\rangle$ is a couple $(V, R)$ where $V$ is a set of (compositional) vectors for the LTSs in $C$ and $A_{\mathrm{ext}}$, and $R$ is a regular expression over $V$.

Example 4. To work our system out, one easily guesses that the client has first to be launched, to connect, the system then runs for some time and finally the client disconnects and exits. This is specified for example using the mapping $\mathrm{M}=\mathrm{v}_{\text {launch }} \cdot \mathrm{v}_{\mathrm{cx}} \cdot \mathrm{M}_{\mathrm{run}} \cdot \mathrm{v}_{\mathrm{dx}} \cdot \mathrm{v}_{\text {exit }}$ with vectors $\mathrm{v}_{\text {launch }}=$ launch?: $\left\langle\right.$ run?, $\varepsilon>, \mathrm{v}_{\mathrm{cx}}=\tau:<\log$ ! id? $>$, $\mathrm{v}_{\mathrm{dx}}=\tau:<$ end!, $\varepsilon>$, and $\mathrm{v}_{\text {exit }}=$ exitCode! $:<$ exitCode!, $\varepsilon>$. Yet, it is more complicated to know what should be done while the system runs $\left(M_{\text {run }}\right)$, excepted of course that events are exchanged for requests/results and that somehow a reset (resending the client identification) should be used. Therefore, one may choose to keep this part of the mapping abstract: $M_{\text {run }}=\left(v_{\text {req }}+v_{\text {res }}+v_{\text {err }}+v_{\text {reset }}\right) *$ with vectors $v_{\text {req }}=$ $\tau:<$ request!,sqlQuery?>, $\mathrm{v}_{\text {res }}=\tau:<$ reply?,sqIValues! $>, \mathrm{v}_{\mathrm{err}}=\tau:<$ reply?,sqIError! $>$, and $\mathrm{v}_{\text {reset }}=\tau:\langle\varepsilon, \mathrm{id} ?>$.

Discussion on the mapping notation. Mappings are made up of the definition of possible correspondences (vectors) and a dynamic description over such correspondences (regex). Several behavioural languages may be used to this purpose. We have presented regex for their simplicity. However, the only requirement for the algorithms presented in Section 2.4 to work is to be able to obtain from the mapping an LTS where transitions are labelled by vectors (Algorithm 1. line 13]). Currently, Adaptor supports both regex and the direct use of LTS. Message Sequence Charts (MSC) where arrows are labelled by vectors are a user-friendly alternative. LTS can be obtained from MSC using, e.g., [24]. We are also investigating the use of techniques from the composition of Web services [6] in order to get automatically possible correspondences between services (vectors) and ease the user task in the context of end-user composition in pervasive systems.

\subsection{Algorithms}

Using a mapping and component behavioural interfaces, an adaptor can be generated automatically for a closed system following results from, e.g., 2114811. Here, our algorithms (Alg. 1 and 2) enable adaptation on open systems. Algorithm 1, works by translating into a Petri net [16] the constraints of a correct adaptor. This choice is done as Petri nets enable to see messages exchanged between components as resources of the adaptor, to de-synchronise messages and 

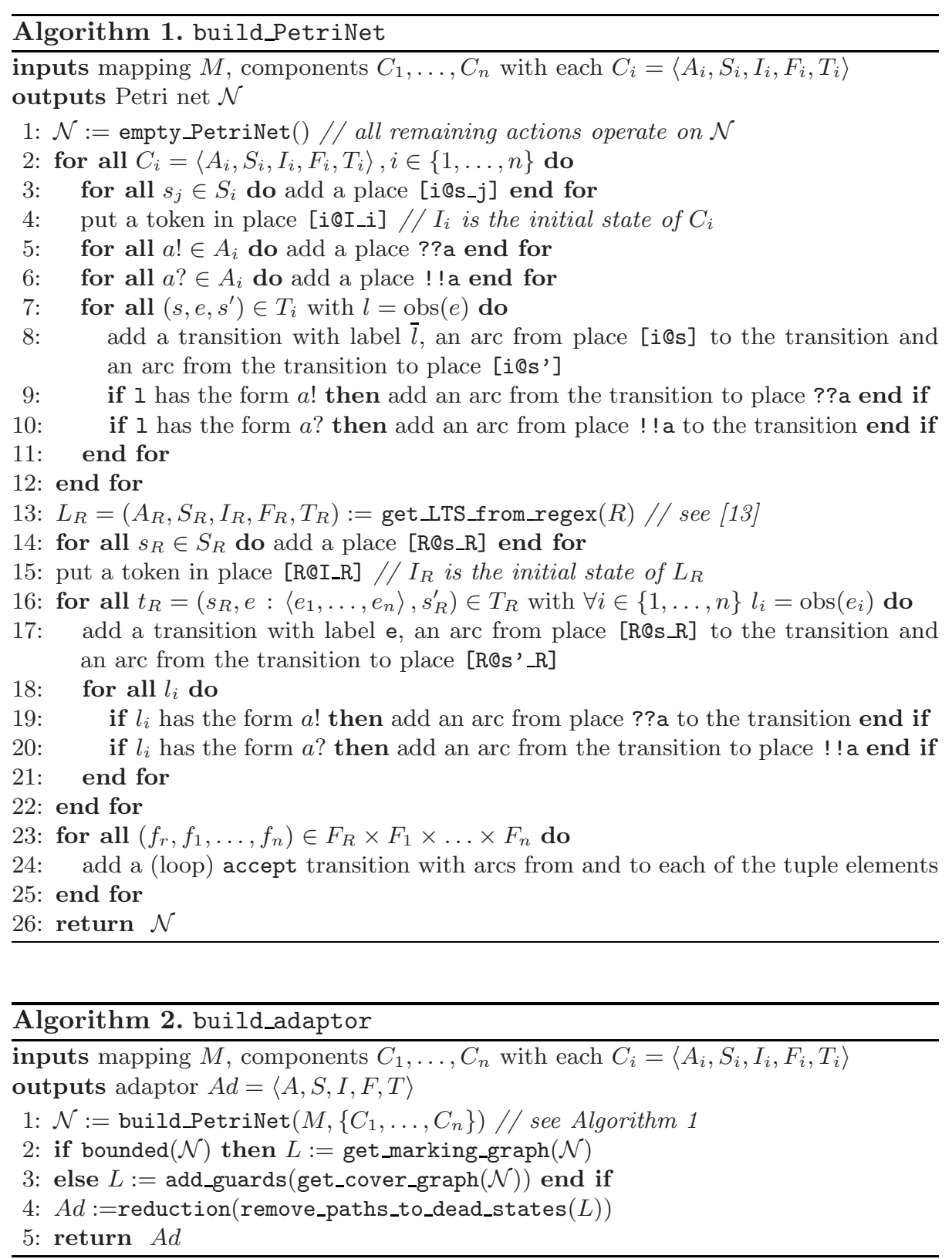

therefore support reordering when required. The encoded adaptor constraints are as follows. First, the adaptor must mirror each component interface (places and transitions are generated from component interfaces, lines 22 12). It must also respect the adaptation contract specified in the mapping (places and transitions are generated, lines 14 22, from an LTS description of the mapping 
obtained in line [13). Algorithm 2 works out the building of the adaptor from this net using several functions. bounded checks if a Petri net is bounded. If so, its marking graph is finite and can be computed (get_marking_graph); if not, then we rely on an abstraction of it, a cover graph (get_cover_graph), where the $\omega$ symbol abstracts any token number $>0$. Due to the over-approximation of cover graphs, add_guards is used on them to add a guard [\#??a>1] (\#??a meaning the number of tokens in place ??a) on any a! transition leaving a state where \#??a is $\omega$. remove_paths_to_dead_states recursively removes transitions and states yielding deadlocks. The optimising of resulting adaptors is achieved thanks to reduction techniques (reduction). Branching reduction [25] is the most appropriate choice as it does not require a strict matching of $\tau$ transitions like strong equivalence. In addition, branching equivalence is the strongest of the weak equivalences, therefore properties restricted to visible actions (e.g., deadlock freedom, but also safety and fair liveness) are preserved by reduction modulo branching equivalence.

Example 5. We present in Figure 2 the Petri net generated for the Client and SQLServer example. To help the reader, we present separately the different parts of the net which are generated for Client (top left), SQLServer (top right) and the mapping (bottom left). The accept transition and the dashed places are used to glue the three subnets. The resulting adaptor is also shown (bottom right). It is more complex than its contract, which demonstrates the need for automatic adaptation processes as presented here.

Our algorithms are supported by Adaptor which relies on ETS [18 for open product computation, TINA [7] for the marking and cover graph computation, and on CADP 12 for adaptor reduction. Due to the computation of marking/cover graphs for the Petri net encodings, this algorithm is in theory exponential in the size of the Petri net, which in turn is related to the sum of the sizes of the component protocols and their alphabets $\left(\sum_{i \in\{1, \ldots, n+1\}}\left(\left|S_{i}\right|+\left|A_{i}\right|\right)\right)$. Yet, in practice, the adapted components are sequential, hence parts of generated Petri nets are 1-bounded which lowers the complexity. The incremental mechanism for adaptation we present in the next section also helps in minimising the complexity of computing adaptors.

\section{Incremental Adaptation of Open Component Systems}

We may now describe an incremental adaptation approach suitable to open systems. At design-time, it helps in the design and integration of component-based systems, grounding on automatic adaptor-connector generation. At run-time, it avoids the computation of global adaptors and supports evolving systems.

\subsection{Architectural Style}

The definition of an architectural style is the support for the description, reasoning and implementation of software architectures. As far as design-time incremental adaptation is concerned, resulting design architectural models will 

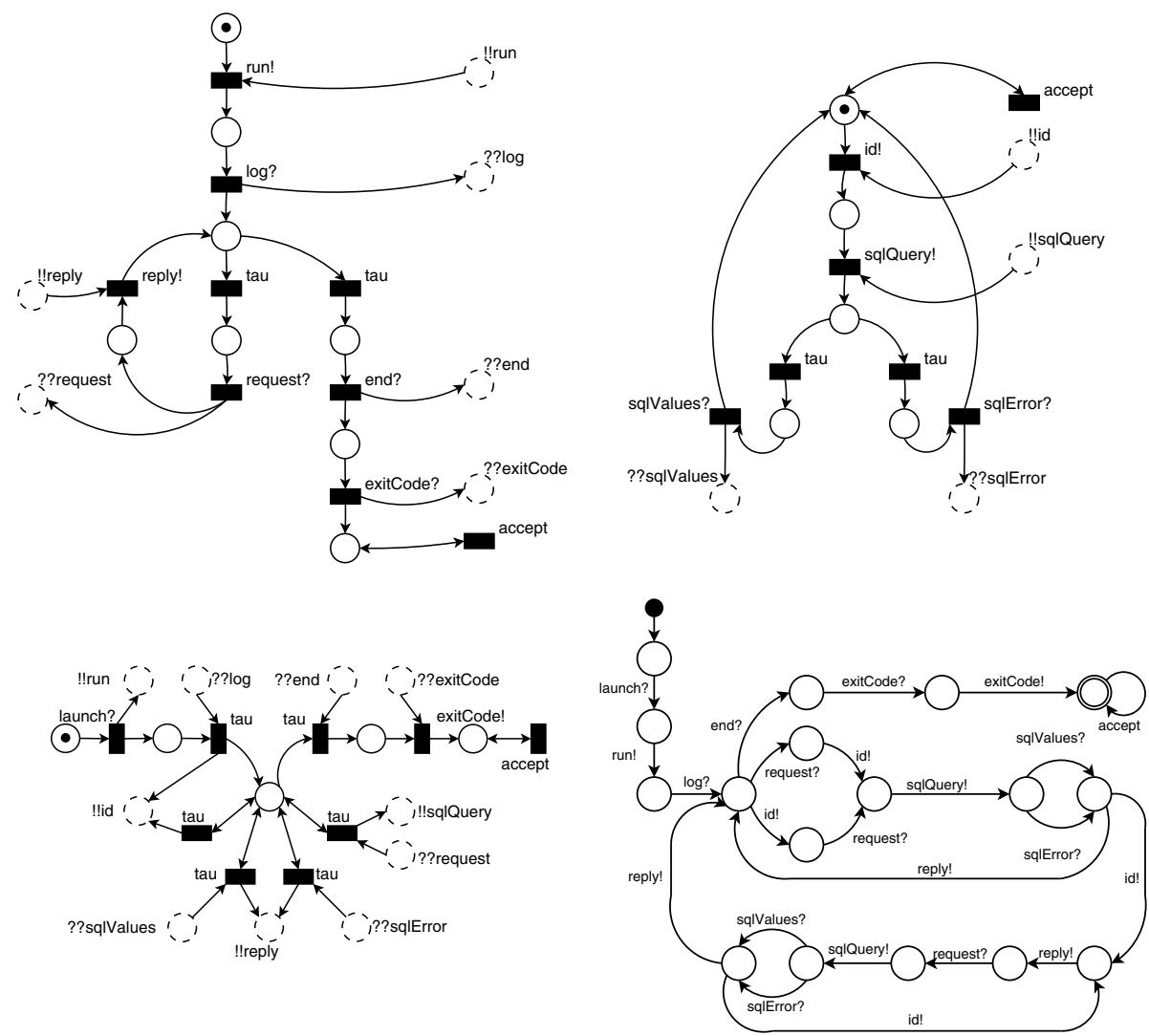

Fig. 2. The Client and Server Example Adaptor Generation

respect our style. As far as run-time incremental adaptation is concerned, communication mechanisms are constrained by it.

Two kinds of entities are distinguished: components and adaptors. Components implement the system's functionalities or services. Adaptors are used as intermediates to avoid deadlock and enforce different coordination policies whose properties are specified in an abstract way in mappings. A component is only connected to its adaptor, and interacts with the rest of the system through it. If the component does not require adaptation, our approach automatically generates a no-op adaptor which reproduces from an external point of view exactly the same behaviour as the component. Adaptors can be connected to other adaptors in order to ensure the system's global correctness.

To support implementation or run-time adaptation, two kinds of interactions have to be distinguished at an adaptor level: with its component and with other adaptors. Adaptors have to agree on a common implementation communication protocol to communicate altogether. Mismatch between components which has been solved thanks to adaptors should not be transferred to a mismatch between 
adaptors which should communicate correctly by construction. Prefixing will help there. Communications with the environment are prefixed by the component identifier and communications with other adaptors are prefixed by the identifier of the components these adaptors are in charge of. As far as communication between adaptors and components is concerned, communications are not prefixed as adaptation should be transparent for the adapted component. The use of prefixing is demonstrated in Section 4 on our application.

\subsection{Assessment}

Adaptors may impose service restriction due to the application of the function removing paths to dead states in the adaptation algorithm (Alg. 2, line 4). These are hard to detect by hand and assessment procedures are therefore required to help the designer (for design-time adaptation) or the end-user (for run-time adaptation). We propose tool-supported procedures based on alphabet comparison and property checking.

Alphabet-based assessment and comparisons may be used either to check the adapted system for services or more specifically to compare the adapted component with reference to the original one. In the first case we may check either successfully synchronised services (obtained hiding in LTSs any transition $e:<l_{1}, \ldots, l_{n}>$ where there is only one $i$ such that $l_{i} \neq \varepsilon$ ) or actions left open to the environment, possibly new services provided by composites (obtained hiding in LTSs any transition $e:<l_{1}, \ldots, l_{n}>$ where there are at least two different $\left.l_{i} \neq \varepsilon\right)$. Comparison between original and adapted components can be performed on the same basis (internal or external comparison) through difference between their respective alphabets.

Property checking is a finer grained technique and may efficiently be used to detect more subtle architectural flaws. Classical properties such as liveness properties (e.g., any request will eventually be satisfied, see Sect. 4 for an application of this) can be easily formalised reusing patterns [15], and then checked against the adapted system model (LTS) using model-checkers. An interesting benefit is that, when the property is unsatisfied, model-checkers return back a counter-example sequence of service calls that may help modifying mappings.

\subsection{Addition and Suppression of Components}

In this section, we present the algorithms for the addition and for the suppression of components. In the addition algorithm (Alg. 3), a component $\left(C_{n+1}\right)$ is adapted and integrated into an existing composite (possibly empty). Adaptation is performed using only the component to be added, a given mapping and adaptors of components referred to in the mapping. Assessment is used to check the result of the integration. It is important to note that, as a preliminary step, automatically built mappings can be proposed. When the system is empty, a no-op mapping, $\left(V,\left(\sum_{v \in V} v\right) *\right)$ with $V=\left\{\left(C_{n+1}: e, e\right) \mid e \in A_{C_{n+1}}\right\}$, simply wraps the added component. When there are already components to communicate with in the system, a trivial mapping, $\left(V,\left(\sum_{v \in V} v\right) *\right)$ with $V=$ 

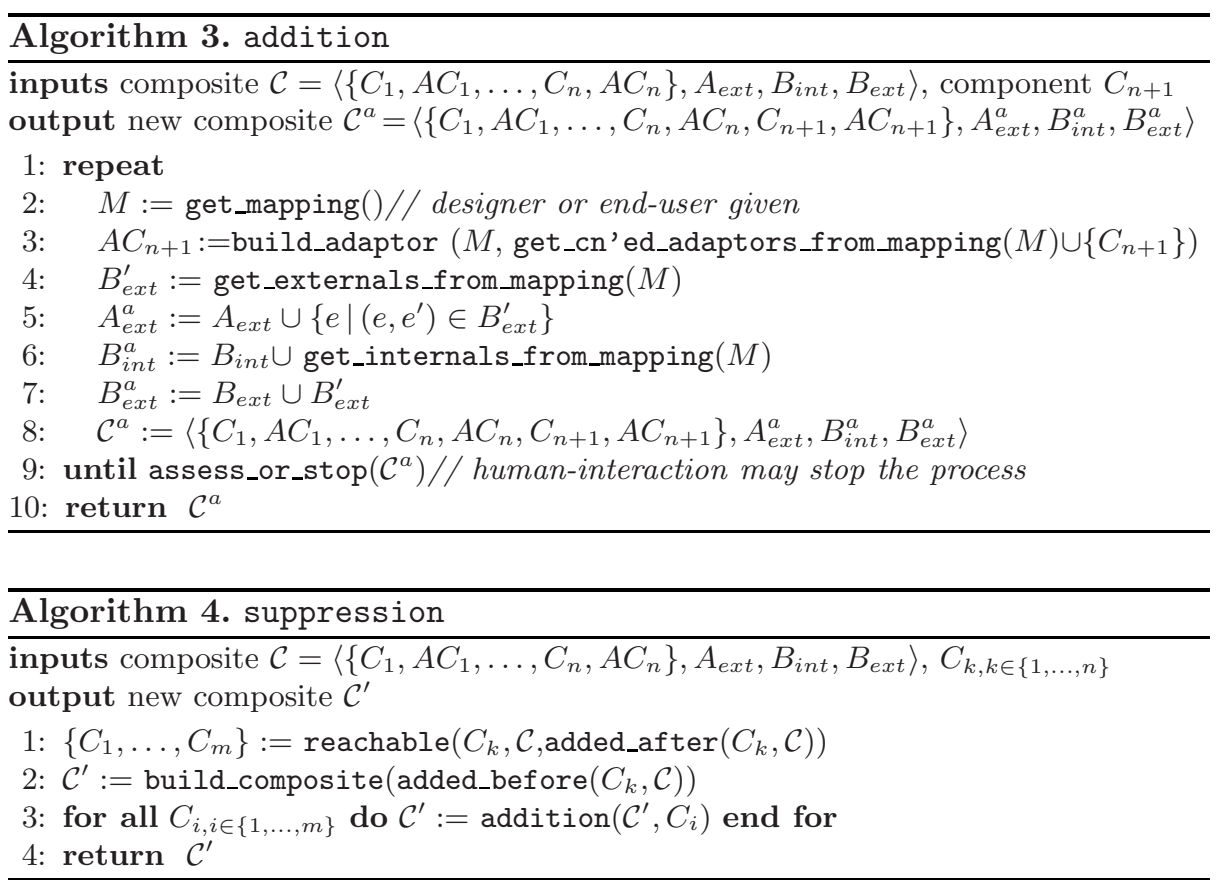

$\left\{\left(C_{i}: e, \bar{e}\right) \mid C_{i}: e \in A_{\text {ext }} \wedge \bar{e} \in A_{C_{n+1}}\right\}$, can be tested. In this algorithm, function get_cn'ed_adaptors_from_mapping iterates over the set of vectors $V$ of the mapping $M$. For each $v$ in $V$, if $v$ respects the form given for $B_{\text {int }}$ in Definition 3 , we can obtain a couple $\left(l_{i}, l_{j}\right)$ and then, looking at the $n$ adaptors alphabets, determine the adaptor $l_{i}$ corresponds to. Function get_externals_from_mapping (resp. get_internals_from_mapping) returns the set of couples $\left(e, l_{i}\right)$ (resp. $\left.\left(l_{i}, l_{j}\right)\right)$ from the vectors $e:\left\langle l_{1}, \ldots, l_{n}\right\rangle$ of the mapping $M$ that respect the form given for $B_{\text {ext }}$ (resp. $B_{\text {int }}$ ) in Definition 3 . The suppression algorithm (Alg. 4) first computes all the components that have been added after the component to be removed, and are reachable (in terms of the architectural graph topology) from it. The suppression may impact all these components, therefore their corresponding adaptors are successively updated if needed using the component addition algorithm. In this algorithm we use the following functions. Function added_after (resp. _before) returns the ordered set of all components of the composite $\mathcal{C}$ added after (resp. before) the component $C_{k}$. Function reachable returns all components of the composite $\mathcal{C}$ present in a given filtering set (added_after results) which are reachable from the component $C_{k}$. Finally, build_composite is used to build a composite applying the addition algorithm on an ordered set of components (result of added_before in the algorithm), and reusing mappings from the former composite construction. Mappings are therefore kept with adaptors while building the system. Removing a component induces the suppression of its adaptor, but also the update of all the adaptors 
interacting with it. In the worst case, this corresponds to recompute all adaptors which is as costly as the regular case in global adaptation approaches where the adaptor is always recomputed.

\section{Application}

We have validated our approach on several examples: the dining philosopher problem, a video-on-demand system, a pervasive music player system, and several versions of a library management application. We present here a simplified version of the latter one. The system manages loans in a library. Components were chosen non recursive (this corresponds to the notion of transactional services) to obtain readable resulting adaptors.

The first component, LIB, tests if a book is available in the library or has been borrowed by a user.

$$
\operatorname{LIB}[i, f]=\text { isBorrowed?. (available!.0 + borrowed!.0) }
$$

A no-op adaptor, ALIB, is first computed using a no-op mapping generated automatically as presented in Section 3.3 . Then, a second component, SUB, is added. It is used as a front-end to the LIB component and checks if a user is a subscriber of the library. If not, SUB replies with the notAvailable! message, otherwise it tests if the requested book is borrowed or available.

$$
\begin{aligned}
& \text { SUB }[i, f]=\text { info?.isRegistered?. (isBorrowed!.SUB_AUX + notAvailable!.0) } \\
& \text { SUB_AUX }=\text { (notBorrowed?.available!.0 + borrowed?.notAvailable!.0) }
\end{aligned}
$$

It is obvious that the components present both name and protocol mismatch, therefore the trivial mapping fails assessment. We recall that events are prefixed except for those corresponding to interactions between the adaptor and its component (see Section 3.1). To work the mismatch out, a mapping $\mathrm{M} 1=$ (v1.v2. (v3+v4. (v5.v6+v7.v3)))* is proposed, with vectors

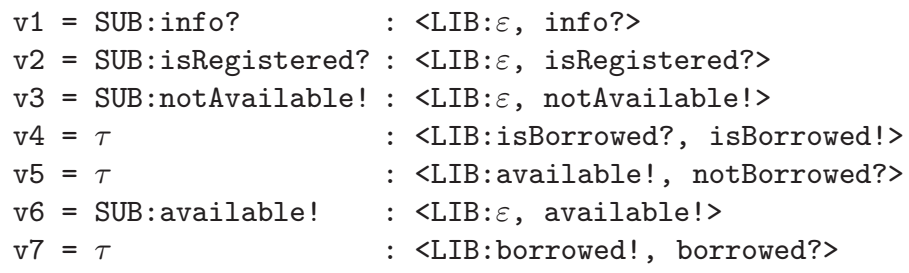

In Figure 3 we present the architecture resulting from our incremental integration and adaptation process. The left hand part is related to the architecture after the addition of SUB and its adaptor, ASUB. The overall figure corresponds to the final architecture (after all components have been added, see BOR below). The architecture is computed automatically using Algorithm 3 .

It was not possible to give all binding names $\left(A_{\text {ext }}, B_{\text {int }}, B_{\text {ext }}\right)$ in the figure due to lack of place. However, bindings here are between ports of same name as the architectures are correct by construction using adaptation. The adaptor ASUB generated from $\mathrm{M} 1$ is shown in Figure 4.

A third component, BOR, receives requests for loans and checks if the book can be borrowed or not (id! stands for identifiers of the user and book). 


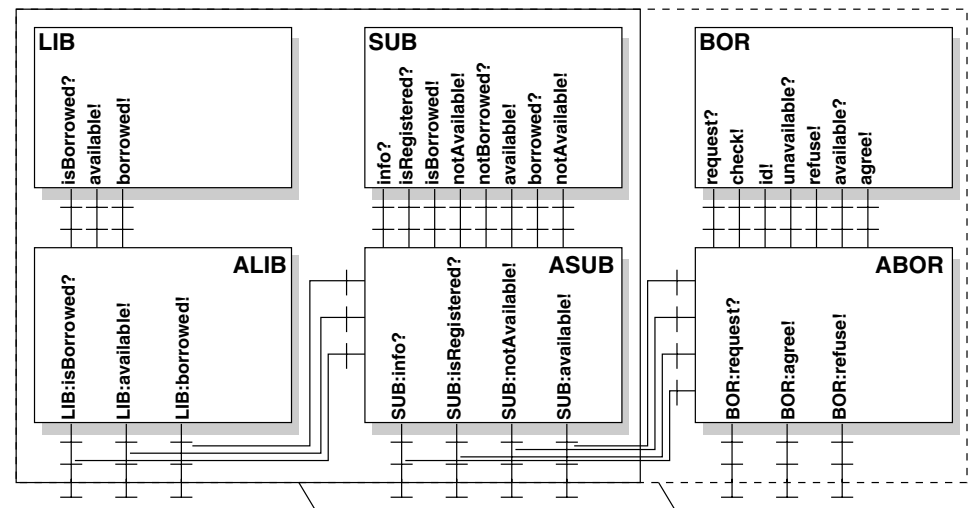

step 2 architecture (after adding SUB) step 3 architecture (after adding BOR)

Fig. 3. The Library Example Architecture

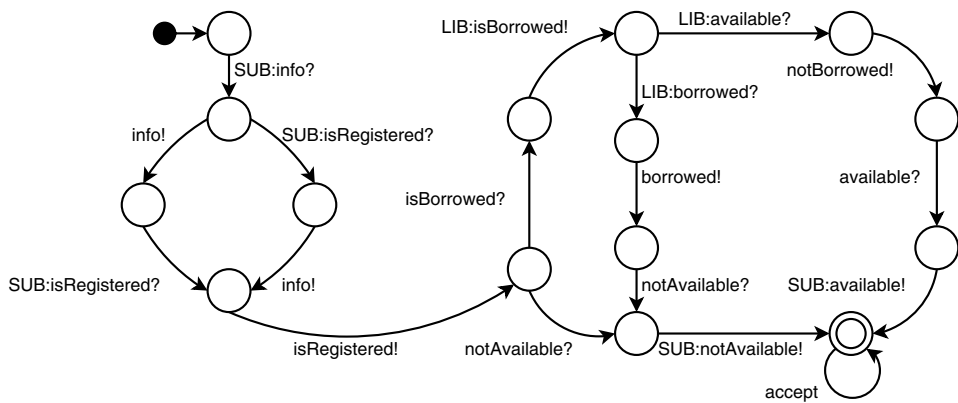

Fig. 4. The Library Example Adaptor

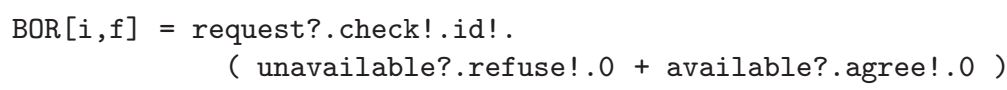

Component BOR can be connected to component SUB using the mapping M2 to make all three components work together. Note in the mapping below that reordering is needed since BOR sends first the check! message and then information about the request id!, whereas SUB accepts first info?, and then the request message isRegistered?. Therefore, the following sequence belongs to the ABOR adaptor: check?.id?.SUB:info! .SUB:isRegistered!.

$$
\mathrm{M} 2=\mathrm{v} 1 \cdot \mathrm{v} 2 \cdot \mathrm{v} 3 \cdot(\mathrm{v} 4 \cdot \mathrm{v} 5+\mathrm{v} 6 \cdot \mathrm{v} 7) *
$$

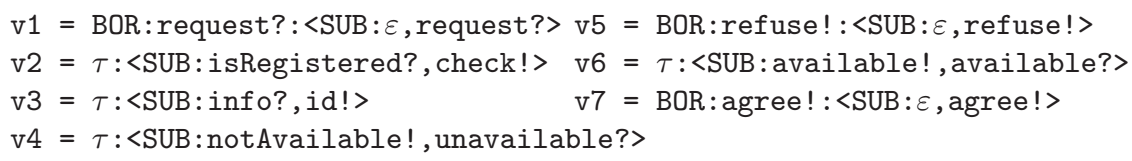

Now, let us illustrate assessment procedures on the system made up of the three components, and their corresponding adaptors. This system is quite simple (33 states, 60 transitions, and 25 labels) since the adaptation process has removed 
all incorrect interactions. External alphabet contains messages BOR:request?, BOR:agree!, BOR:refuse!, and also all the messages left observable in the previous steps, i.e., LIB: isBorrowed?, LIB: available!, etc. The synchronised alphabet contains all the remaining messages which are connected internally. In addition, the system is deadlock-free and verifies the following liveness property:

[true*] (["BOR:request?"]

(mu X. (<true> true and [not ("BOR:agree!" or "BOR:refuse!")]X)))

It states that messages BOR:request! are always followed after a finite number of steps either by a message BOR:agree? or BOR:refuse?. Basically, this means that all requests are always replied, which corresponds to the classic pattern "AG request $\Rightarrow \mathrm{EF}$ reply" encoded in $\mu$-calculus. This property was automatically checked using Evaluator the model-checker of CADP. Consequently, the BOR adaptor is validated, and the final correct architecture is as presented earlier on.

Let us now remove component SUB. This can be done for update purposes or just because the loan check is simplified not to take into account that the user has to be a subscriber. BOR is the only connected component added after SUB. A new mapping is given for component BOR to connect it directly to component LIB, M2' = v1.v2.v3. (v4.v5+v6.v7)* with vectors

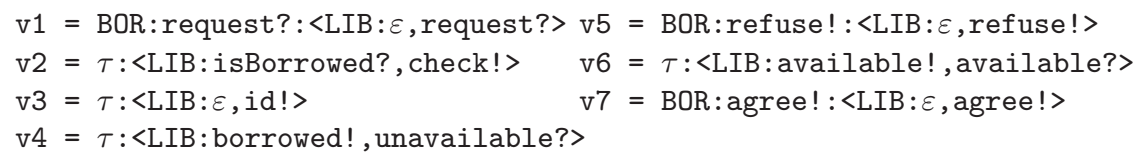

The corresponding adaptor is computed, the new system assessed successfully, and we end up with a system made up of components LIB, BOR, and their respective adaptors. To check how the approach integrates in a complete development process, the system has been implemented in COM/DCOM using the adaptor models to obtain their code.

\section{Related Work}

Since Yellin and Strom's seminal paper 27, adaptation techniques 21148 have been proposed to correct component mismatch building adaptors. In [1] we made significant advances with an approach supporting name mismatch, systemwide adaptation (more than two components) and event reordering. Yet, all these approaches require the computation of a global adaptor, which is costly, and none supports open systems, which prevents application to pervasive systems.

In 22, component wrappers are composed to augment connector behaviour. This has been revisited in [23], providing automation, but still with a centralised global adaptor as starting point, as for 4] where adaptor distribution is addressed. Several theoretical works have focused on the incremental construction of systems and dynamic reconfiguration $5,3,26$. However, these proposals only address syntactic adaptation (via name translation or morphisms) and cannot be used to solve behavioural mismatch. In [19], we have proposed a methodology to help designers in the incremental construction of componentbased systems where adaptors are required. The definition of open systems, their 
composition and related adaptation algorithms were not supported. Incrementality was achieved using implicit vectors exporting in adaptors all the component services in order to make the design process compatible with adaptation as defined in [11. This limits the application of [19] at design-time where the set of components to be integrated is known.

\section{Conclusion}

The integration of software components often requires a certain degree of adaptation. Adaptation approaches have addressed closed systems and the distribution of global adaptors but to our knowledge, none supports open systems. Thus, they are not well suited to systems where components or services may enter and leave at any time, such as pervasive ones. To address this issue, we have proposed here (i) a formalising of component-based open systems which thereafter supports, (ii) an extension of software adaptation to open systems, and (iii) an incremental integration process which avoids the computation of global adaptors. The adaptation solutions we propose are supported by a tool, Adaptor. In its current version, Adaptor can deal with both closed systems and open systems. This tool and its set of validation examples (approx. 70 examples, 25,000 lines of XML specifications) are freely available from [1].

Our main perspectives concern the application of our model-based adaptation techniques to service oriented architectures for pervasive computing. First, relations between adaptation models and implementation languages have to be studied. We have done some experiments using COM/DCOM but Web services are more relevant in this area. The combination of adaptation with semantic composition solutions such as [6] is also an interesting perspective to support not only behavioural but also semantic correctness. To end, end-user composition is a crucial issue in pervasive computing. The support in Adaptor for the use of other adaptation contract formalisms, as presented in Section 2.3 is therefore an interesting perspective.

Acknowledgements. This work has been supported by the French National Network for Telecommunication Research. Adaptor has been developed with S. Beauche. We thank M. Tivoli for the COM/DCOM encoding of the case study and C. Canal for fruitful discussions.

\section{References}

1. The Adaptor tool (LGPL licence). Available from P. Poizat's Webpage

2. Agha, G.: Special Issue on Adaptive Middleware. CACM 45(6), 30-64 (2002)

3. Aguirre, N., Maibaum, T.: A Logical Basis for the Specification of Reconfigurable Component-Based Systems. In: Pezzé, M. (ed.) FASE 2003. LNCS, vol. 2621, Springer, Heidelberg (2003)

4. Autili, M., Flammini, M., Inverardi, P., Navarra, A., Tivoli, M.: Synthesis of Concurrent and Distributed Adaptors for Component-based Systems. In: Gruhn, V., Oquendo, F. (eds.) EWSA 2006. LNCS, vol. 4344, Springer, Heidelberg (2006) 
5. Back, R.J.: Incremental Software Construction with Refinement Diagrams. Technical Report 660, Turku Center for Computer Science (2005)

6. Ben Mokhtar, S., Georgantas, N., Issarny, V.: Ad Hoc Composition of User Tasks in Pervasive Computing Environments. In: Gschwind, T., Aßmann, U., Nierstrasz, O. (eds.) SC 2005. LNCS, vol. 3628, Springer, Heidelberg (2005)

7. Berthomieu, B., Ribet, P.-O., Vernadat, F.: The tool TINA - Construction of Abstract State Spaces for Petri Nets and Time Petri Nets. International Journal of Production Research, vol. 42(14), 2741-2756 (2004)

8. Bracciali, A., Brogi, A., Canal, C.: A Formal Approach to Component Adaptation. Journal of Systems and Software 74(1), 45-54 (2005)

9. Bruneton, E., Coupaye, T., Leclercq, M., Quéma, V., Stefani, J.-B.: The Fractal Component Model and Its Support in Java. Software Practice and Experience, vol. 36(11-12), 1257-1284 (2006)

10. Canal, C., Murillo, J.M., Poizat, P.: Software Adaptation. L'Objet. Special Issue on Software Adaptation 12(1), 9-31 (2006)

11. Canal, C., Poizat, P., Salaün, G.: Synchronizing Behavioural Mismatch in Software Composition. In: Gorrieri, R., Wehrheim, H. (eds.) FMOODS 2006. LNCS, vol. 4037, Springer, Heidelberg (2006)

12. Garavel, H., Lang, F., Mateescu, R.: An Overview of CADP 2001. EASST Newsletter 4, 13-24 (2002)

13. Hopcroft, J.E., Ullman, J.D.: Introduction to Automata Theory, Languages and Computation. Addison Wesley, London, UK (1979)

14. Inverardi, P., Tivoli, M.: Deadlock Free Software Architectures for COM/DCOM Applications. Journal of Systems and Software 65(3), 173-183 (2003)

15. Manna, Z., Pnueli, A.: Temporal Verification of Reactive Systems: Safety. Springer, Heidelberg (1995)

16. Murata, T.: Petri Nets: Properties, Analysis and Applications. Proceedings of the IEEE 77(4), 541-580 (1989)

17. Objet Management Group. Unified Modeling Language: Superstructure. version 2.0, formal/05-07-04 (August 2005)

18. Poizat, P.: Eclipse Transition Systems. RNRT project STACS deliverable (2006)

19. Poizat, P., Salaün, G., Tivoli, M.: An Adaptation-based Approach to Incrementally Build Component Systems. In: Proc. of FACS'06 (2006)

20. Satyanarayanan, M.: Pervasive Computing: Vision and Challenges. IEEE Personal Communications 6(8), 10-17 (2001)

21. Schmidt, H.W., Reussner, R.H.: Generating Adapters for Concurrent Component Protocol Synchronization. In: Proc. of FMOODS'02, Kluwer Academic Publishers, Dordrecht (2002)

22. Spitznagel, B., Garlan, D.: A Compositional Formalization of Connector Wrappers. In: Proc. of ICSE'03, ACM Press, New York (2003)

23. Tivoli, M., Autili, M.: SYNTHESIS, a Tool for Synthesizing Correct and ProtocolEnhanced Adaptors. L'Objet. 12(1), 77-103 (2006)

24. Uchitel, S., Kramer, J., Magee, J.: Synthesis of Behavioural Models from Scenarios. IEEE Transactions on Software Engineering 29(2), 99-115 (2003)

25. van Glabbeek, R.J., Weijland, W.P.: Branching Time and Abstraction in Bisimulation Semantics. Journal of the ACM 43(3), 555-600 (1996)

26. Wermelinger, M., Lopes, A., Fiadeiro, J.L.: A Graph Based Architectural (Re)configuration Language. In: Proc. of ESEC/FSE'01, ACM Press, New York (2001)

27. Yellin, D.M., Strom, R.E.: Protocol Specifications and Components Adaptors. ACM Transactions on Programming Languages and Systems 19(2), 292-333 (1997) 